\title{
EXISTENCE OF CLOSED TIMELIKE GEODESICS IN LORENTZ SPACES ${ }^{1}$
}

\author{
FRANK J. TIPLER
}

\begin{abstract}
Certain classes of compact four-dimensional Lorentz spaces are shown to possess at least one closed timelike geodesic.
\end{abstract}

1. Introduction. The problem of existence of closed geodesics on a Riemannian manifold is one of the most fundamental questions in global differential geometry [1]. Existence of closed geodesics has been established for compact Riemannian manifolds, but no work has been done on establishing existence in compact Lorentz manifolds, possibly because it has been felt that existence proofs require a definite metric. In this paper a beginning is made toward extending existence theory of closed geodesics to compact Lorentz manifolds. Specifically, it is shown that in an important class of four-dimensional compact Lorentz manifolds-those with a covering space containing a compact Cauchy surface-closed timelike geodesics exist. My notation and conventions will be those of [2]. In particular, a spacetime $(M, g)$ is a $C^{\infty}$ boundaryless four-dimensional connected Hausdorff manifold $M$ with a $C^{\infty}$ Lorentz metric $g$. $(M, g)$ is time-orientable and orientable. All Cauchy surfaces $S$ are boundaryless acausal imbedded submanifolds of $M$ such that $D(S)=M$; i.e., every inextendible causal curve in $M$ intersects $S$ exactly once.

2. THE THEOREM. Let $(M, g)$ be a compact spacetime with a covering space containing a compact Cauchy surface. Then $(M, g)$ contains a closed timelike geodesic.

Proof. Let $(\tilde{M}, \tilde{g})$ denote the covering space to $(M, g)$ which contains a compact Cauchy surface. Geroch [3, p. 444] (see also [2, p. 212]) has shown that $\tilde{M}$ is homeomorphic to $\tilde{S} \times \mathbf{R}$, where $\tilde{S}$ is the Cauchy surface, and that all Cauchy surfaces in $(\tilde{M}, \tilde{g})$ are compact. Since $(M, g)$ is a compact spacetime, it must contain a closed timelike curve $\gamma[2$, p. 189]. Let $\tilde{\gamma} \subset \tilde{M}$ be

Received by the editors November 3, 1978.

AMS (MOS) subject classifications (1970). Primary 53C50, 53C05; Secondary 54D30, 53B25, $53 \mathrm{C} 40$.

Key words and phrases. Lorentz manifolds, closed geodesics, compact manifolds, covering space, global hyperbolicity, global analysis.

${ }^{1}$ Research supported by the National Science Foundation under grant number PHY-77-15191. 
a timelike curve segment which is mapped one-to-one and onto $\gamma$ by the covering map $f$. The closure of $\tilde{\gamma}$ has two endpoints, $\tilde{p}^{\prime}$ and $\tilde{p}^{\prime \prime}$, both of which are mapped into the same point of $\gamma$ by the covering map. By the compactness of $\tilde{S}$, the fact that $\tilde{M}$ is homeomorphic to $\tilde{S} \times \mathbf{R}$, and the properties of the covering map, there exist disjoint compact Cauchy surfaces $\tilde{S}^{\prime}$ and $\tilde{S}^{\prime \prime}$ such that $\tilde{p}^{\prime} \in \tilde{S}^{\prime}, \tilde{p}^{\prime \prime} \in \tilde{S}^{\prime \prime}$, and $\tilde{S}^{\prime}$ is isometric to $\tilde{S}^{\prime \prime}$. (Pick any compact Cauchy surface $\tilde{S}^{\prime}$ through $\tilde{p}^{\prime}$, and use the covering map to construct its image $\tilde{S}^{\prime \prime}$ through $\tilde{p}^{\prime \prime}$. The Cauchy surfaces $\tilde{S}^{\prime}$ and $\tilde{S}^{\prime \prime}$ will be disjoint by the properties of the covering map.) The distance function $d\left(\tilde{u}^{\prime}, \tilde{v}^{\prime \prime}\right)$ for all points $\tilde{u}^{\prime} \in \tilde{S}^{\prime}$ and $\tilde{v}^{\prime \prime} \in \tilde{S}^{\prime \prime}$ is finite and continuous in $\tilde{u}^{\prime}$ and $\tilde{v}^{\prime \prime}$ by Lemma 6.7 .3 of [2, p. 215]; in particular it is finite and continuous for corresponding points $\tilde{p}^{\prime}, \tilde{p}^{\prime \prime}$ of $\tilde{S}^{\prime}$ and $\tilde{S}^{\prime \prime}$, respectively. Since $d\left(\tilde{p}^{\prime}, \tilde{p}^{\prime \prime}\right)$ is a continuous function on the compact set $\tilde{S}^{\prime \prime}$, it attains its maximum value for the points $\tilde{q}^{\prime}, \tilde{q}^{\prime \prime}$ say. By Proposition 6.7.1 of [2, p. 213] and the argument on p. 216 of [2], there exists a timelike geodesic $\tilde{\alpha}$ of length $d\left(\tilde{q}^{\prime}, \tilde{q}^{\prime \prime}\right)$ between $\tilde{q}^{\prime}$ and $\tilde{q}^{\prime \prime}$. The geodesic $\tilde{\alpha}$ projects into a closed timelike curve $\alpha$ in $(M, g)$, and $\alpha$ is a geodesic everywhere except possibly at $f\left(\tilde{q}^{\prime}\right)=f\left(\tilde{q}^{\prime \prime}\right)=q$, where the tangent to $\alpha$ might not be defined. However, $\alpha$ must be a geodesic with well-defined tangent at $q$ also, for otherwise by Proposition 4.5.3 of [2, p. 105] we could deform $\alpha$ into a closed timelike curve whose cover in $(\tilde{M}, \tilde{g})$ would have greater length from $\tilde{S}^{\prime}$ to $\tilde{S}^{\prime \prime}$ than $\tilde{\alpha}$. But this is impossible since $\alpha$ has maximal length among all timelike curves from a point in $\tilde{S}^{\prime}$ to the corresponding point in $\tilde{S}^{\prime \prime}$. Thus $\alpha$ is a closed timelike geodesic in $(M, g)$.

3. Discussion. The above theorem can be extended without much difficulty to any dimension greater than or equal to 2 , since all the necessary lemmas from [2] can easily be generalized to any dimension $\geqslant 2$. I have restricted the proof given above to four dimensions in order to simplify the proof.

The restriction to compact Lorentz manifolds with globally hyperbolic covering spaces is unfortunately very severe. Such a restriction is necessary in order to use without modification the distance function theory for Lorentz manifolds developed by Hawking [2]. However, the above theorem does apply to most simple examples of Lorentz manifolds. The restriction to nonsimply connected compact Lorentz manifolds is not strong, at least in four dimensions, since in four dimensions all compact Lorentz manifolds are not simply connected [2, p. 190]. It is hoped that the ideas used in the proof of the above theorem could suggest a proof for general Lorentz manifolds.

It should be mentioned that no compact Lorentz manifold is of any physical interest, since all such manifolds have closed timelike curves $([2, \mathrm{p}$. 189], [4, p. 38], [5, p. 783]).

\section{REFERENCES}

1. W. Klingenberg, Lectures on closed geodesics, Grundlehren der Mathematischen Wissenschaften, Band 230, Springer-Verlag, Berlin, 1978. 
2. S. W. Hawking and G. F. R. Ellis, The large-scale structure of spacetime, Cambridge Univ. Press, Cambridge, 1973.

3. R. P. Geroch, The domain of dependence, J. Mathematical Phys. 11 (1970), 437-449.

4. R. Penrose, Techniques of differential topology in relativity, SIAM, Regional Conference Series in Applied Mathematics, no. 7, 1972.

5. R. P. Geroch, Topology in general relativity, J. Mathematical Phys. 8 (1967), 782-786.

Department of Mathematics, University of California, Berkeley, California 94720 